\title{
ENTROPIC \\ SPACETIME \\ THEORY
}




\section{SERIES ON KNOTS AND EVERYTHING}

Editor-in-charge: Louis H. Kauffman

Published:

Vol. 1: Knots and Physics

L. H. Kauffman

Vol. 2: How Surfaces Intersect in Space

J. S. Carter

Vol. 3: Quantum Topology

edited by L. H. Kauffman \& R. A. Baadhio

Vol. 4: Gauge Fields, Knots and Gravity

J. Baez \& J. P. Muniain

Vol. 5: Gems, Computers and Attractors for 3-Manifolds

S. Lins

Vol. 6: Knots and Applications

edited by L. H. Kauffman

Vol. 7: Random Knotting and Linking edited by $K$. C. Millett \& D. W. Sumners

Vol. 8: Symmetric Bends: How to Join Two Lengths of Cord R. E. Miles

Vol. 9: Combinatorial Physics

T. Bastin \& C. W. Kilmister

Vol. 10: Nonstandard Logics and Nonstandard Metrics in Physics W. M. Honig 
IfI Series on Knots and Everything - Vol. 13

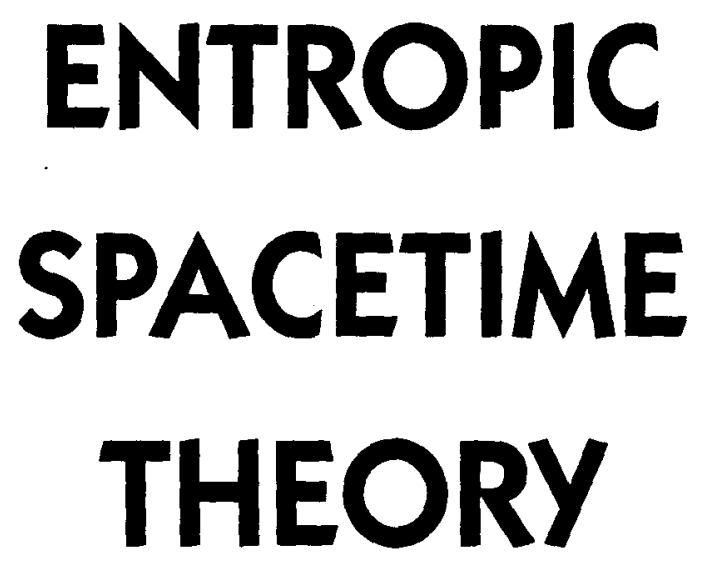

\section{Jack Armel}

The Foundation for Physical Sciences

USA 


\title{
Published by
}

World Scientific Publishing Co. Pte. Ltd.

P O Box 128, Farrer Road, Singapore 912805

USA office: Suite 1B, 1060 Main Street, River Edge, NJ 07661

UK office: 57 Shelton Stret, Covent Garden, London WC2H 9HE

\section{Lbrary of Congress Cataloging-in-Publication Data \\ Armel, Jack.}

Entropic spacetime theory / Jack Armel.

128 p. $22 \mathrm{~cm}$.- (Series on knots and everything : 13)

Includes index.

ISBN 9810228422

1. Space and time. 2. Entropy. I. Title. II. Series: K \& E series on knots and everything : vol. 13.

QC173.59.S65A865 1996

$530.1-\mathrm{dc} 20$

96-28661

CIP

\section{British Library Cataloguing-in-Publication Data}

A catalogue record for this book is available from the British Library.

\author{
Copyright 1996 The Foundation for Physical Sciences \\ 770 South Palm Avenue, Suite 1102-1103 \\ Sarasota, FL 34236, USA
}

This book is printed on acid-free paper.

Printed in Singapore by Uto-Print 


\section{ENTROPIC SPACETIME THEORY -A COMMENT \\ by Louis $\mathrm{H}$. Kauffman}

The purpose of this short comment on Jack Armel's Entropic Spacetime Theory (EST) is to bring out, in skeletal form, the major assumptions of the theory and how these assumptions work together.

First, it is assumed as given the de Broglie formula $E=h \omega$ where $\omega=c / \lambda, h$ is Planck's constant, $c$ is the speed of light, $\lambda$ denotes wavelength and $E$ is energy. It is assumed that the de Broglie formula applies to the energy of space itself.

Second, it is postulated that there is a quantum of space with minimal length $l_{0}$, and that to each (quantum of) energy there is an associated distance l. This distance $l$ is an integral multiple of $l_{0}$.

Third, energy is stored in space according to Hooke's law: $E=k l$. (The constant $k$ is determined by constraints of the theory).

In this context of space quanta, de Broglie and Hooke, it is natural to associate a wavelength $\lambda$ with a length $l: l=\lambda x$. What is $x$ ? We have $E=h \omega=h c / \lambda=h c x / l$, and we have $E=k l$. Thus $k l=h c x / l$. Hence $x=k l^{2} / h c$ and we conclude that for $\lambda$ the wavelength of a space-quantum of length $l, \lambda l=h c / k$. Wavelength is inversely proportional to physical length with a constant of proportionality $h c / k$.

Now we assume that the minimal length $l_{0}$ corresponds to the minimal energy $E_{0}=h$. Thus $h=E_{0}$ implies $\omega_{0}=1$. This implies $\lambda_{0}=c$. Hence $l_{0} \lambda_{0}=h c / k$ becomes $l_{0} c=h c / k$. Thus $l_{0}=h / k$. The minimal length is the ratio of Planck's constant and the spring constant.

Now consider the energy density $\rho_{0}=E_{0} / l_{0}{ }^{3}$ associated with the space quantum $l_{0}$. We have $\rho_{0}=E_{0} /(h / k)^{3}=h /(h / k)^{3}=k^{3} / h^{2}$. Planck's constant being very small, the energy density of the EST vacuum is very high! See the text to see just how high this density turns out to be. This high vacuum energy density is one of the key points of EST. Space itself becomes the final reference for all energetics.

So far we have made no assumptions about the geometrical nature of the space-quantum, other than its associated length $l$ and elastic ability to store energy. EST further assumes a geometric model for these quanta as small chunks of spacetime. Model building for more complex quanta such as photons, electrons and quarks proceeds geometrically and topologically under the constraints imposed by known facts and parameters about these entities. Many remarkable fittings occur at the submicroscopic and cosmological levels. 
This page is intentionally left blank 


\section{PREFACE}

Even if there is no end in the search of knowledge, I awakened this morning with a sense of completion. The last page was in place, the giant crossword puzzle was filled in...the entropic spacetime theory was as complete as I was capable to make it. Please don't laugh when I tell you that the morning after completing this work I started on two new aspects of this theory which I felt were not yet properly looked after. What kind of a theory is it?

It is a theory of space. It suggests how and when all matter and radiation was created. It proposes a cosmology. It delineates what "dark matter" is. It describes a discrete universe and offers specific dimensional limits.

It preserves the conservation of energy in all its aspects; there is no need to abandon it in cosmology. It has order-it cycles from expansion (where we are today) to an equilibrium, than to a compressive epoch. The universe never reached the state of "singularity" as many of our brothers have envisioned. The cosmos just "breathes" like we do.

The destiny of humanity is not forever, but we are likely to exist long enough to attain great ends. The projected length of our existence in this universe is about twice the age of the present epoch. That is, we should exist through the equilibrium and again as long as the universe is old now. The equivalent of some forty billion years of the projected future seems quite ample.

Yes, there was a big bang, the theory proclaims and there will be another and another in due course. The bang will be caused by matter-antimatter release of energy. Like the seasons, each new cycle will carry some of the previous seasons surviving galaxies into the new cycle. In other words each new epoch will start expanding fully equipped with the elements of the galaxies. An expanding epoch cannot create new galaxies from space. That is why our astronomers cannot place the creation of galaxies into our present epoch.

But then this theory does not deal with cosmology exclusively. It actually deals with the nature of space. I only told you about the future of our universe so that you will say-"It sounds all right but how did you figure this out?" 
viii

You will be surprised, as I was, when you find that space is very dense-more dense than any matter can be. You will be less surprised when I tell you that this theory says that time is variable and is not much good for measuring the passing of events for really long periods.

"How is it then that you gave predictions in billions of years?" You might say.

Well it happens that most of the expansion has already taken place $(85 \%)$ in only half of the expected duration of the universe, before compression starts. Moreover the compression epoch starts very slowly and speeds up later. So if you believe that our present age is already some 20 billion years old (our time) then we will have another twenty billion before compression starts and a comparable twenty billion more in the next epoch-the one where antimatter is being created. In other words time crawls in tiny increments in the early stages after the bigbang. From now until the corresponding point in the compression epoch which resembles ours, time is not quite so ridiculous and can be approximated to units which we understand.

I have another surprise for you, before you ask how is matter or antimatter being created. In this theory, space, (called entropic spacetime) is the creator of all elementary things: radiation and matter. It can only do that in the compressive epoch.

There are other surprises in this theory. The universe is discrete and finite. Everything-not only energy comes in little bundles. Length, charge and time are all quantized.

Another surprise is that space is not a void or a vacuum but consists of tiny electric charges (a ten billionth the size of the electron). These charges are both positive and negative and are called dipoles. The distance between the charges is time and length. (Spacetime in effect). The energy content of space spreads the charges apart and hence we have expansion of space.

This makes it possible to explain gravity.

This is a highly deterministic theory-just the opposite of everything you've been accustomed to hearing about physics. There are numbers and values for all these items. There is no infinity, except in mathematics-and that is one of humankind's exclusive inventions which is not taken directly from observed nature. No, it does not attack or destroy the present physics of relativity or quantum theory. It sits in between the two, because it deals exclusively with space as a thing. It deals mostly with "rest energy" not kinetic energy. The latter is left to the present physics.

This covers the "WHAT". If you want to find out "HOW" then you'll have to read on.

Would you believe that it took more than thirty years to figure this out? The 
last ten rather intensively! I must be grateful to Helen for putting up with me for such a stretch. Also my son David, who calls me regularly to ask how the "great work" is coming along. Most of all I am grateful to Lou Kauffman, the professor and knot theorist from the University of Illinois, who could easily pass as my son. He has challenged and inspired me all at once and for a long time. 
This page is intentionally left blank 


\section{CONTENTS}

PREFACE v v

CHAPTER I An Overview of the Theory 1

An approach to space $\quad 3$

A cure for infinity 4

Conservation of energy 5

Quantization of space $\quad 6$

Dilation 7

CHAPTER II The Fabric of Entropic Spacetime 9

$\begin{array}{lr}\text { A quantitative description of space } & 10\end{array}$

$\begin{array}{ll}\text { Constant of elasticity } & 13\end{array}$

Description of space 13

$\begin{array}{ll}\text { CHAPTER III Gravity } & 17\end{array}$

Gravitational attraction-why not repulsion? 18

The interface between EST and the kinetic system 20

The acceleration of gravity from entropic data 20

The Mach Principle 21

Causality 23

Gravitons 24

Density of EST space $\quad 24$

Energy content 24

Time 24

Conversion of conventional density to EST density 26

How does gravity work? 27

Buoyancy analogy 27

Antigravity 28

CHAPTER IV Cosmology 29

A vast mass of entropic spacetime 29

One Friedmann universe $\quad 30$

Singularity 31

Temperatures $\quad 32$

Equilibrium data on space quanta 33

The age problem $\quad 34$

After the entropic equilibrium 34 
At equilibrium 36

Touching on quantum field theory 36

About matter collapsing into high densities $\quad 37$

The end of the contraction epoch 38

Peering at the edge of time 38

More on when the galaxies were created $\quad 39$

A rationale for dark matter $\quad 39$

A review on the entropic spacetime theory 41

Internal gravitational force of space quanta $\quad 42$

On Great Attractors, the Wall, the Great Void 42

The upper limit 43

The dimensions of the universe $\quad 44$

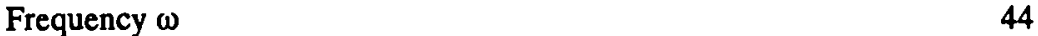

Reconciling methods $\quad 46$

CHAPTER V The Inflationary Universe Theory-Is It Necessary? 47

The horizon problem $\quad 47$

Where are we today? $\quad 50$

Gravitational lens bending $\quad 51$

Time 51

What happens to time at the big bang part of cycle? 52

Black holes? 53

CHAPTER VI Model Making of Radiation and Matter in a Shrinking Universe $\quad 55$

Model 1. The smallest vector boson $\quad 56$

Model 2. A beam of photons $\quad 57$

Model 3. Vector boson capable of pair production $\quad 57$

Model 4. An electron $\quad 58$

Model 5. Phase change 1 59

Phase 2. A writhe $\quad 60$

Phase 3. A writhe of writhes $\quad 61$

A conceptual quark $\quad 61$

Specification-quark $\quad 62$

Neutrons and protons $\quad 62$

Bosons and fermions $\quad 64$

The neutrino $\quad 66$

Model making and quantum mechanics $\quad 67$

Double slit experiment—Wave Particle duality 68

$\begin{array}{ll}\text { Why a photon knows no time } & 70\end{array}$

$\begin{array}{ll}\text { Time and velocity in a discrete universe } & 71\end{array}$

$\begin{array}{ll}\text { CHAPTER VII Surfaces } & 73\end{array}$

The Coulomb law breakdown $\quad 75$

$\begin{array}{ll}\text { More on surfaces } & 76\end{array}$ 
The classical radius of the electron

Upon the anomaly of the electron magnetic moment 78

The anomaly, as seen through EST Theory 78

CHAPTER VIII EST and the Quantum Field Theory 81

Outside the standard model $\quad 82$

A little more on quantum theory 83

The analogy with Moiré sheets $\quad 84$

CHAPTER IX Unitless Identities of Nature $\quad 85$

Conversion of entropic value to unitless identity. 86

$\begin{array}{ll}\text { Empiricals } & 87\end{array}$

The Planck equations $\quad 87$

Tests $\quad 89$

Planck mass $\quad 91$

CHAPTER X Forces 93

The strong force $\quad 93$

The weak force 94

The gravitational force $\quad 95$

The electrical force 95

Other derivations $\quad 96$

Summary $\quad 98$

CHAPTER XI Laws of EST and a Few Proofs 101

Second Law 101

Is there an interaction between matter and EST? 102

A note about E 104

More "Proofs"

APPENDIX 1
Wavelength Ranges for Scalar Bosons, Vector Bosons and Fermions 107

TABLES OF EST VALUES 108

INDEX 113 